\title{
FUSION OF MULTI-FOCUS IMAGES WITH NeIGHBOUR LOCAL DisTANCE
}

\author{
Ias Sri Wahyuni ${ }^{1}$ and Rachid Sabre ${ }^{2}$ \\ ${ }^{1}$ Universitas Gunadarma, Jl. Margonda Raya No. 100 Depok 16424, Indonesia \\ ${ }^{2}$ Laboratory Biogéosciences CNRS, \\ University of Burgundy/Agrosup Dijon, France
}

\begin{abstract}
The aim of multi-focus image fusion is to integrate images with different objects in focus so that we obtained a single image with all objects in focus. In this paper, we present a novel multifocus image fusion method based on neighbour local variability (NLV). This method takes into consideration the information in the surrounding region of pixels. Indeed, at each pixel, the method exploits the local variability calculated from quadratic difference between the value of pixel and the value of all pixels that belong to its neighbourhood. It expresses the behaviour of pixel relative to all pixels belonging to its neighbourhood. The variability preserves edge feature because it detects the abrupt image intensity. The fusion of each pixel is performed by weighting each pixel by the exponential of the local variability. The precision of this fusion depends on the largenumberof the neighbourhood where the largenumber depends on the blurring characterized by the variance and its size of blurring filter. We constructed a model that gives the value of the large.... from the variance and the size of blurring filter. Comparing our method with other methods, it shows the best result.
\end{abstract}

\section{KEYWORDS}

Neighbour Local Variability; Multi-focus image fusion; Root Mean Square Error (RMSE)

\section{INTRODUCTION}

Due to the limited depth-of-focus of optical lenses, it is often difficult to capture an image that contains all relevant objects in focus. Only the objects within the depth-of-field are in focus, while other objects are blurred. Multi-focus image fusion is developed to solve this problem. There are various approaches that have been performed in literature. These approaches can be divided into two types: the spatial domain method and the multi-scale fusion method. The spatial domain fusion method is performed directly on the source images. In spatial domain techniques, we directly deal with the image pixels. The pixel values are manipulated to achieve the desired result. The fusion methods such as averaging, Principal Component Analysis (PCA) [1], maximum selection rule, bilateral gradient-based methods [2] and Guided Image Filter (GIF)based method [3] and maximum selection rule fall under spatial domain approaches. The disadvantage of spatial domain approaches is that they produce spatial distortion in the fused image. Spatial distortion can be very well- handled by multi-scale approaches on image fusion. In multi-scale fusion methods, the fusion process is performed on the source images after decomposing them into multiple-scales. The discrete wavelet transform (DWT) [4]-[9], Laplacian pyramid image fusion [10]-[17], Discrete cosine transform with variance calculation (DCT+var) [18], saliency detection based method (SD)[19] are examples of image fusion techniques under transformdomain. 
In this paper, we propose pixel level multi-focus image fusion based on the neighbour local variability (NLV). This method takes into consideration the information of the surrounding region of pixels. Indeed, at each pixel, the method exploits the local variability calculated from quadratic difference between the value of pixel and the value of all pixels that belong to its neighbourhood. It expresses the behaviour of pixel relative to all pixels belonging to its neighbourhood. The variability preserves edge feature because it detects the abrupt image intensity. The fusion of each pixel is performed by weighting each pixel by the exponential of the local variability. The precision of this fusion is depending on the width of region of pixels considered in the neighbourhood. Firstly, we studied the optimal width of region for having the minimum error. Hence, we showed that the width of region depends on the blurring characterized by the variance and its size of blurring filter. We constructed a model that gives the value of the large from the variance and the size of the blurring filter.

While comparing our method with other methods existed in literature (DWT and LP-DWT), it was shown that our method gave the best result by using Root Mean Square Error (RMSE). In this work, the experimental for fusion image and compare to other methods.

This paper is organized as follows: The first section reveals the steps of the fusion process of the proposed method and a model giving the size of neighbourhood. In section 3, we studied the experimental resultsand compared our method to some recent methods. Section 4 gives conclusion of this work. In section 5, we give mathematical details for showing a propriety of the local variability.

\section{THE PROPOSED METHOD}

Consider the fusion of two images, $I_{1}$ and $I_{2}$ that have respectively blurred parts $B_{1}$ and $B_{2}$. These images have the same size: $N_{1} \times N_{2}$. We study the case where $B_{1}$ and $B_{2}$ are disjoint. The idea of the NLV fusion method consists of summing the pixel values of the two images weighted by local variability in each picture. This local variability at $(x, y)$ is calculated from the exponential of average of the square difference between the value of the pixel $(x, y)$ and the value of its neighbors. The NLV at $(x, y)$ is defined as follows:

$$
v_{a, k}(x, y)=\sqrt{\frac{1}{R} \sum_{m=-a}^{a} \sum_{n=-a}^{a}\left|I_{k}(x, y)-I_{k}^{\prime}(x+m, y+n)\right|^{2}}
$$

where $k$ is the index of $k^{\text {th }}$ source image $(k=1,2)$, a is the size of neighborhood

$$
\begin{gathered}
I_{k}^{\prime}(x+m, y+n)=\left\{\begin{array}{l}
I_{k}(x+m, y+n), \text { if } 1 \leq x+m \leq N_{1} \text { and } 1 \leq y+n \leq N_{2} \\
I_{k}(x, y), \text { otherwise }
\end{array},\right. \\
R=(2 a+1)^{2}-\operatorname{card}(S), \\
S=\left\{(m, n) \in\left([-a, a]^{2}-\{(0,0)\}\right) \mid I_{k}^{\prime}(x+m, y+n)=I_{k}(x, y)\right\} .
\end{gathered}
$$

In the annex 1 ,it is shown that this local variability is small enoughwherethe location is on theblurred area $\left(B_{1}\right.$ or $\left.B_{2}\right)$. 
In this paper, we develop a novel fusion method that consists of weighting each pixel of each image by exponential of neighbour local variability. This neighbour local variability is calculated from the quadratic difference between the value of the pixel and the all pixel values of its neighbours. The idea came from the fact that the variation of the value in blurred region is smaller than the variation of the value in focused region. We used the neighbour, with the size "a", of a pixel defined as follows:

$$
(x+i, y+j) \text { where } i=-a,-a+1, \ldots, a-1, a \text { and } j=-a,-a+1, \ldots, a-1, a .
$$

For example, the neighbor with the small size $\left(" a^{\prime \prime}=1\right)$ contains: $(x-1, y-1),(x-1, y)$, $(x-1, y+1),(x, y-1),(x, y+1),(x+1, y-1),(x+1, y),(x+1, y+1)$.

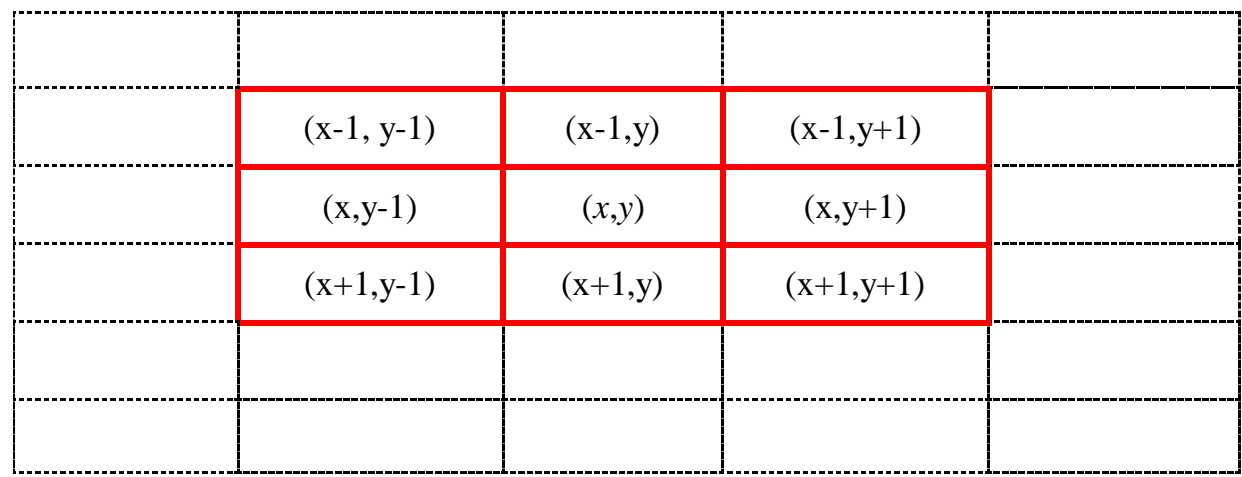

Fig. 2. Pixel at $(x, y)$ within its neighborhood, $a=1$.

Then, the steps of image fusion with size " $a$ " are as follows:

Suppose there are $M$ original source images, $I_{1}, \ldots, I_{M}$, with different focus to be fused. The images here have the same size $\left(N_{1} \times N_{2}\right)$. The general principle of making fusion rules are:

Step 1:For each pixel of each image, we calculated the neighbor local variability (NLV) of every source image, $v_{a, k}(x, y)$ defined in (1).

Step 2: The fused image proposed, $F$ is calculated in the following model:

$$
F(x, y)=\frac{\sum_{k=1}^{M} \exp \left(v_{a, k}(x, y)\right) I_{k}(x, y)}{\sum_{i=1}^{M} \exp \left(v_{a, k}(x, y)\right)}(17)
$$

Obviously, this method depends on the size " $a$ ". First, we tried with a small size $(a=1)$. Hence, the NLV method is better than DWT method. To improve this method and to compare it with all other methods, we optimized the value of " $a$ "for having the minimum Root Mean Square Error (RMSE), where RMSE is defined in section 4. For that, we showed that the value of " $a$ " depends on the blurred area.

The choice of the size of the neighborhood " $a$ " used in NLV method depends on variance $(v)$ and the size $(s)$ of the blurring filter. Our problem is to have a model that gives the value of the " $a$ " according to the " $v$ " and "s"; we take a sample of 1000 images that we blurred using Gaussian filter with different values of $v$ and $\mathrm{s}(v=1,2,3, \ldots, 35$ and $s=1,2,3, \ldots, 20)$. 
After that, for each image we blurred with parameters " $v "$ and " $s "$, we applied our fusion method with different values of " $a "(" a=1,2, \ldots, 17 ")$ and determined the value of " $a$ " that gives the minimum RMSE, denoted by $a_{I}(v, s)$. Then, we took the mean of the $a_{I}(v, s)$ for 1000 images, denoted $a(v, s)$, because the coefficient of variation is smaller than 0.1 .

To propose a model, firstly, we have studied the variation of " $a$ " in according to variance " $v$ " for each fixed size of blurring filter "s". We noted that this variation is logarithmic.For example, $" s=8$ " on Fig. 4.By using nonlinear regression, we obtained the model:

$$
\mathrm{a}=2.1096 \ln v+2.8689 \text {. }
$$

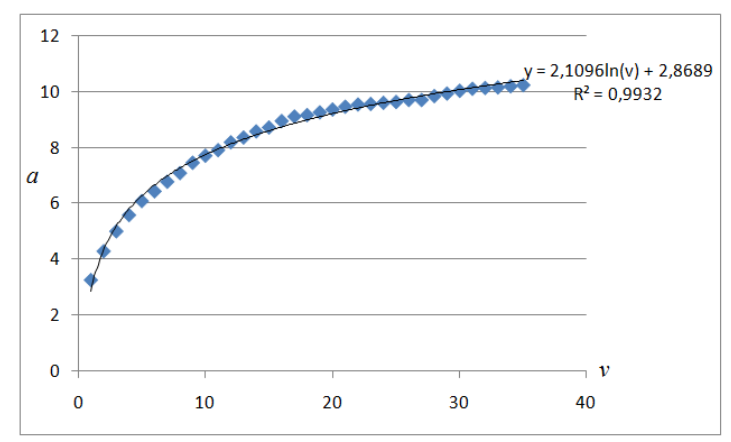

Fig. 4. Graph between "a" and variance of blurring filter where "s"=8.

In general, the model is:

$$
a=c_{1}(s) \ln v+c_{2}(s)
$$

where the $c_{1}$ and $c_{2}$ are functions that depend on "s". The graphs that describe $c_{1}$ and $c_{2}$, respectively, are revealed in Fig. 5. and Fig. 6.
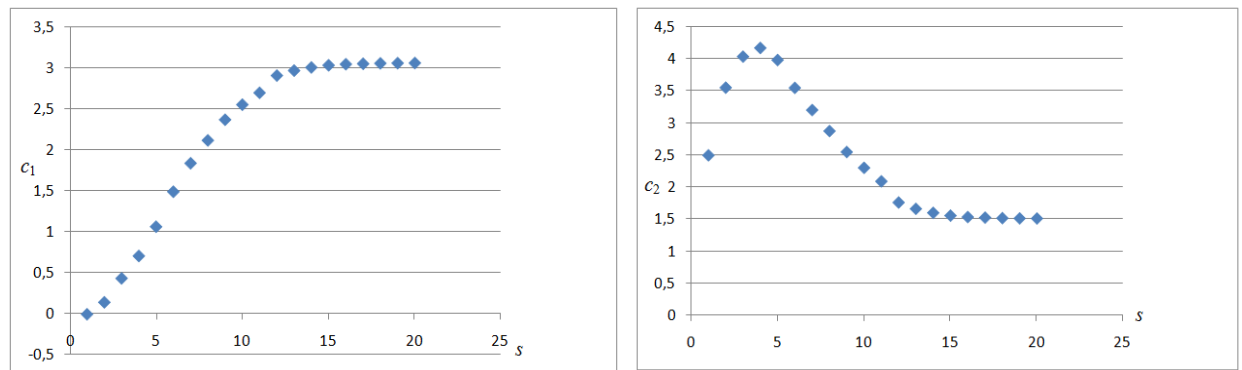

Fig. 5. graph of $c_{1}(s)$ Fig. 6. graph of $c_{2}(s)$.

By giving a model of $c_{1}$ and a model of $c_{2}$ and introducing these models in (19), we get the general following model:

$$
a(v, s)=\frac{3.0348761}{1+29.0909139 \exp (-0.5324955 s)} \ln (v)+0.434\left(\frac{75.062269}{1.225175 s}\right) \exp \left(-0.5\left(\frac{\log (s)-2.655551}{1.225175}\right)^{2}\right)
$$


As " $a$ " is integer, we have two choices of $a$. It is either the floor of $a(v, s)$, denoted by $\lfloor a(v, s)\rfloor$ or the ceiling of $a(\mathrm{v}, \mathrm{s})$, denoted by $\lceil a(v, s)\rceil$ where $\lfloor x\rfloor=\min \{n \in \square \mid n \geq x\}$ and $\lceil x\rceil=\max \{m \in \square \mid m \leq x\}$. Since the RMSE values of both " $a$ " are very slightlydifferent, then we can choose any " $a$ " of them. We use " $a "=\lfloor a(v, s)\rfloor$ in the remaining part of this paper.

We validated our model by applying it to 100 images (we generated 100 pairs multi-focus images with various values of variance and size of blurring filter) and the result is as good as it was expected. Thus, our method is better than DWT and LP-DWT methods. To use this NLV method, we must firstly estimate the variance and the size of blurring filter. For that,there exists some papers giving the methods to estimate variance of blurring filter and the blur detection as in [23][27].We also proposed another method wherein we combined Laplacian pyramid method and NLV method. Indeed, we used Laplacian pyramid with NLV as a selection rule, denoted by LPNLV.

\section{EXPERimental ReSUlt}

The NLV method is performed on a datasets of images [26] using Matlab2013a. We blur these images using Gaussian filter with many values of variance and size. To lighten the reading of the paper, we presented only two examples with the size 256x256 (N1 = N2 = 256). The first, image 'bird' Fig.1 and the second image 'bottle' Fig.2, all images consist of two images with different focus and one reference image.

For comparison purposes, we performed fusion using methods: PCA method [1], Discrete Wavelet Transform (DWT) method [6], Laplacian Pyramid LP_PCA [15], LP_DWT [17] and Bilateral gradient (BG) [2].

In order to compare these methods, we used the following four evaluation criteria frequently used:

\section{Root Mean Square Error (RMSE)}

RMSE finds out the difference between the reference image $\mathrm{R}$ and the fused image $\mathrm{F}$. It gives the information how the pixel values of fused image deviate from the reference image. RMSE between the reference image and the fused image is computed as:

$$
R M S E=\sqrt{\frac{1}{r c} \sum_{i=1}^{c} \sum_{j=1}^{c}[R(x, y)-F(x, y)]^{2}}
$$

where $R$ is a reference image, $F$ is a fused image, $r \mathrm{x} c$ is the size of the input image, and $x, y$ represents to the pixel locations. A smaller value of RMSE shows a good fusion result. If the value of RMSE is 0 then it means the fused image is as exactly the same as the reference image.

For two images that are presented in this paper and blurred with variance $=10$ and size of blurring filter $=5$, the model (20) gives the neighbour size "a" $=5$ and "a" $=6$. Here, we use "a" = 6 because it results the smaller RMSE compared to "a" $=5$ however the RMSE values of "a" $=5$ and $" \mathrm{a} "=6$ are very slightly different. 


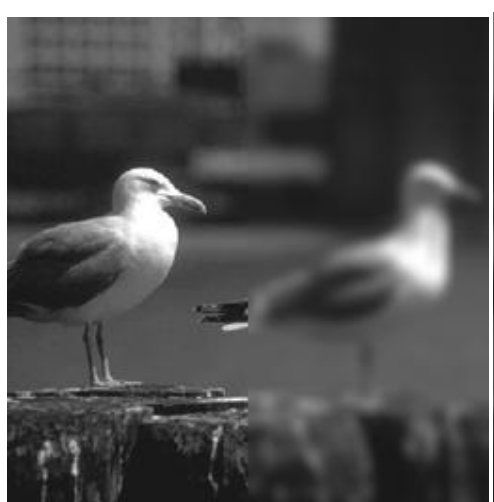

Blurred image 1

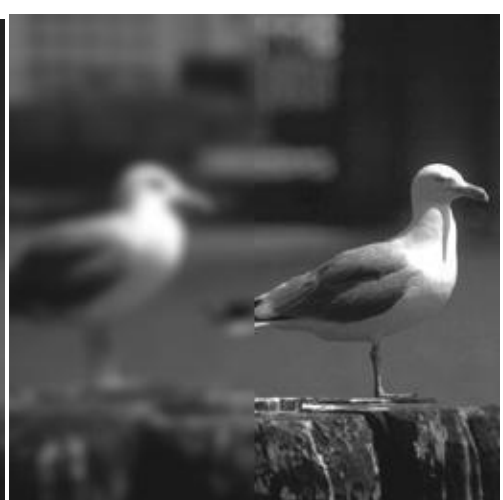

Blurred image 2

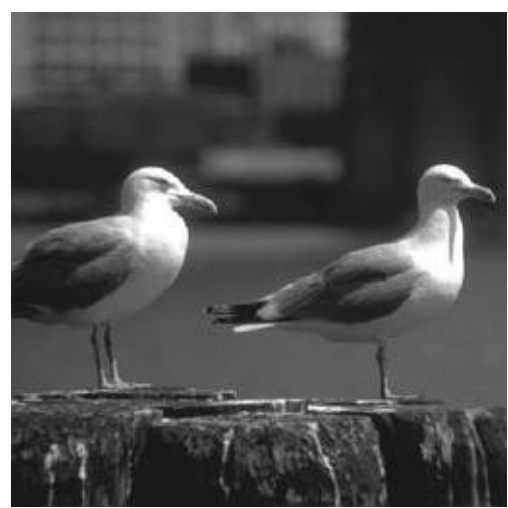

Figure1. Fusion by proposed method NLV

We have found that the NLV method better fusion compared to other methods, see Fig.1.

Table 1. Performance evaluation of image 'bird'

\begin{tabular}{|c|c|c|c|c|c|c|c|c|c|c|}
\hline & PCA & DWT & $\begin{array}{c}\text { LP- } \\
\text { DWT }\end{array}$ & $\begin{array}{c}\text { LP- } \\
\text { PCA }\end{array}$ & DCT+var & $\begin{array}{c}\text { Bilateral } \\
\text { gradient }\end{array}$ & GIF & SD & NLV & $\begin{array}{c}\text { LP- } \\
\text { NLV }\end{array}$ \\
\hline RMSE & 6.9205 & 3.5678 & 1.5190 & 1.4681 & 2.6860 & 8.8378 & 2.2792 & 10.4547 & $\mathbf{0 . 5 4 6 6}$ & 0.8431 \\
\hline
\end{tabular}

From the value of RMSE calculated for ten methods on Table 1, for image'bird': the smallest is NLV method, the second smallest is LP-NLV, the third is LP-PCA, as we can see on the Table 1. NLV method is the best method among the above methods and LP-NLV is better than LP-PCA and LP-DWT.

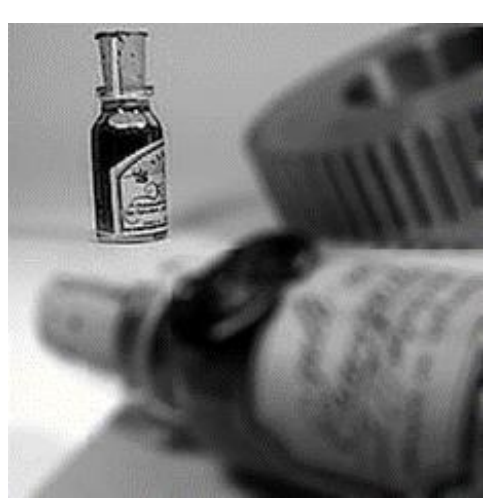

Blurred image 1

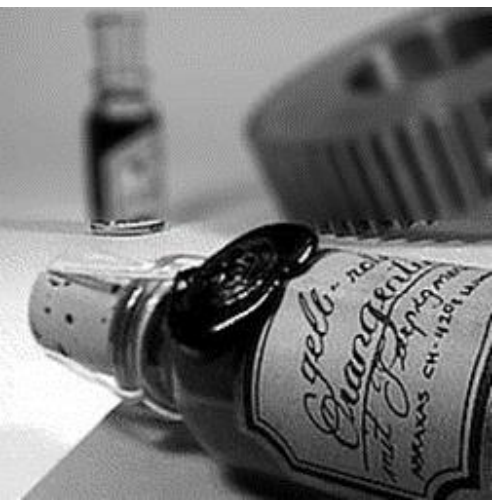

Blurred image 2 


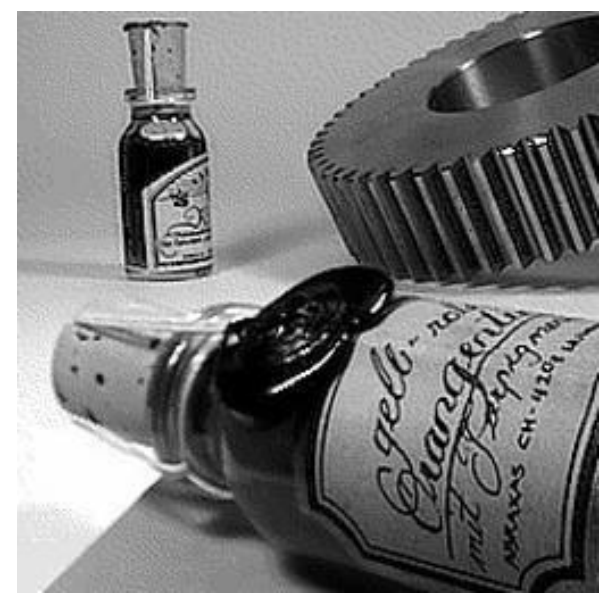

Figure2. Fusion by proposed method (NLV)

We have found that the NLV method performs better compared to other methods, see Fig.2.

To confirm our visually result, we calculated the evaluation metrics: RMSEsee Table 2. From the value of RMSE calculated for ten methods in Table 2, we can classify these methods from the smaller value of RMSE. The smallest value is NLV, the second smallest is LP-NLV, the third smallest is LP-PCA.

Table 2. Performance evaluation of images of 'the bottle'

\begin{tabular}{|c|c|c|c|c|c|c|c|c|c|c|}
\hline & PCA & DWT & $\begin{array}{c}\text { LP- } \\
\text { DWT }\end{array}$ & $\begin{array}{c}\text { LP- } \\
\text { PCA }\end{array}$ & DCT+var & $\begin{array}{c}\text { Bilateral } \\
\text { gradient }\end{array}$ & GIF & SD & NLV & LP-NLV \\
\hline RMSE & $\begin{array}{c}15.00 \\
5\end{array}$ & 5.384 & 2.528 & 2.485 & 2.642 & 20.380 & $\begin{array}{c}3.6 \\
81\end{array}$ & $\begin{array}{c}16.91 \\
9\end{array}$ & $\mathbf{0 . 9 0 2}$ & 1.584 \\
\hline
\end{tabular}

According to the evaluation measure RMSE, the Table 3 gives the mean and standard deviation of RMSE for the considered methods applied on 150 images.

Table 3. Statistic parameters of the sample (150 images)

\begin{tabular}{|c|c|c|c|c|c|c|c|c|}
\hline Methods & $\begin{array}{c}\text { PC } \\
\text { A }\end{array}$ & DWT & $\begin{array}{c}\text { LP_DW } \\
\text { T }\end{array}$ & LP_PCA & $\begin{array}{c}\text { DCT_va } \\
\text { r }\end{array}$ & BG & NLV & LP_NLV \\
\hline Mean & $\begin{array}{c}8,71 \\
3\end{array}$ & 4,194 & 2,049 & 1,995 & 2,839 & 11,044 & 0,591 & 1,344 \\
\hline $\begin{array}{c}\text { Standard } \\
\text { deviation }\end{array}$ & $\begin{array}{c}3,86 \\
6\end{array}$ & 1,381 & 0,756 & 0,743 & 1,308 & 4,859 & 0,204 & 0,697 \\
\hline $\begin{array}{c}\text { Time of } \\
\text { execution } \\
\text { by image }\end{array}$ & $7 \mathrm{~s}$ & $5 \mathrm{~s}$ & $7 \mathrm{~s}$ & $7 \mathrm{~s}$ & $6 \mathrm{~s}$ & $6 \mathrm{~s}$ & $5 \mathrm{~s}$ & $7 \mathrm{~s}$ \\
\hline
\end{tabular}

The results show that the proposed method (NLV) has a smaller mean of the RMSE. The histograms of RMSE for 150 images by different methods (Figures 3, 4, 5, 6, 7, 8 and 9) show for almost all methods that the values of RMSE are almost symmetrically centred around the mean value. 


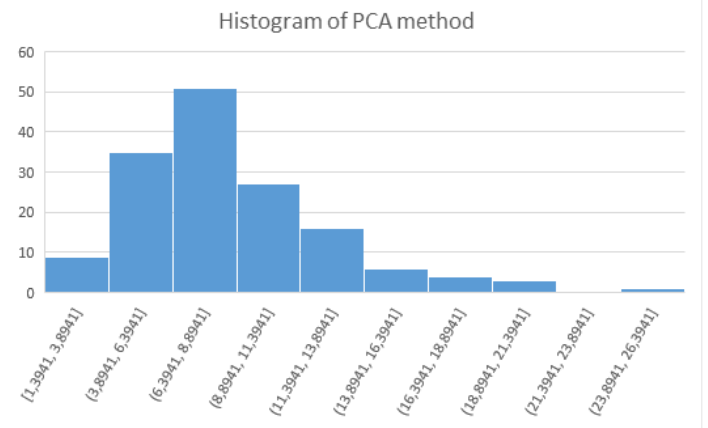

Figure 3. Histogram of PCA method

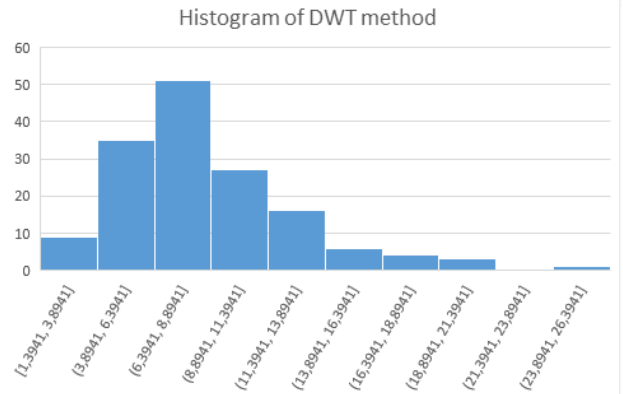

Figure 5. The Histogram of DWT method

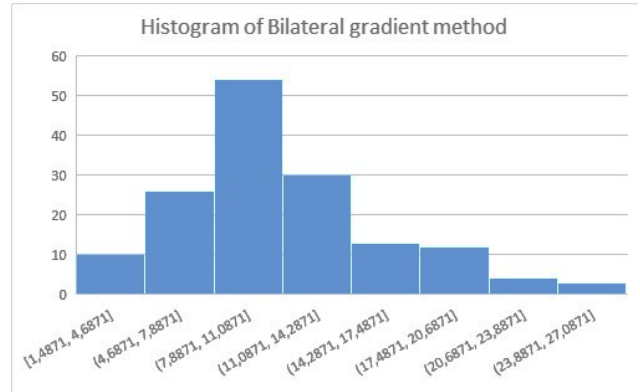

Histogram of LP_PCA

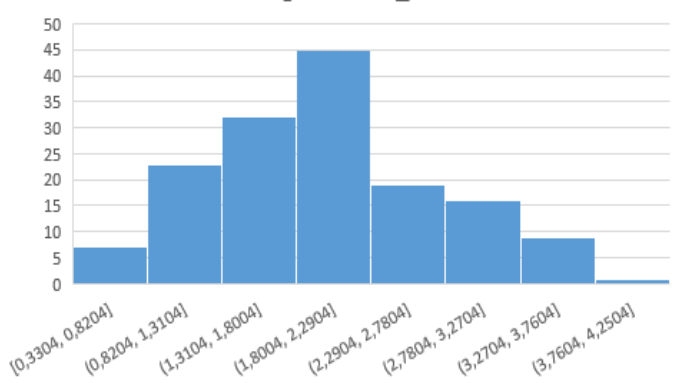

Figure 4. Histogram of LP_PCA method

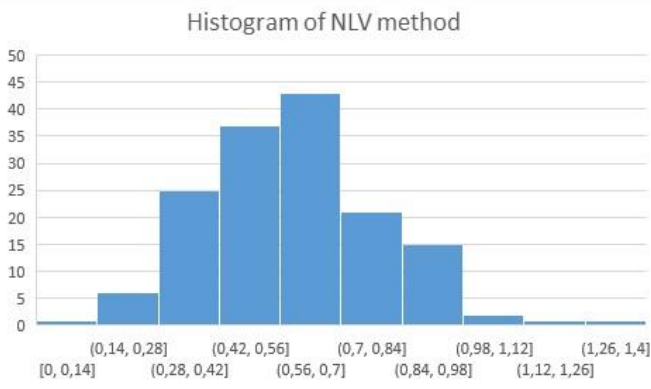

Figure 6. Histogram of LP_DWT method

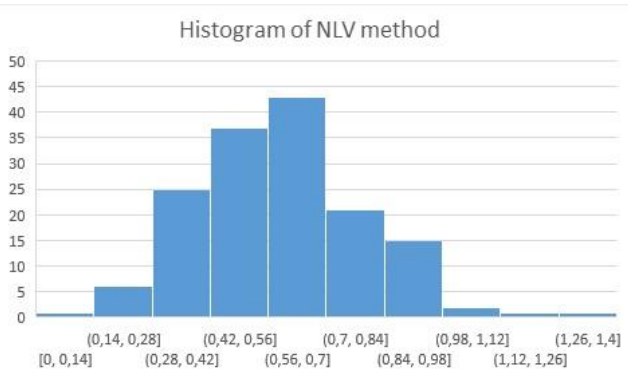

Figure 7. Histogram of Bilateral gradient method Figure 8. Histogram of NLV method

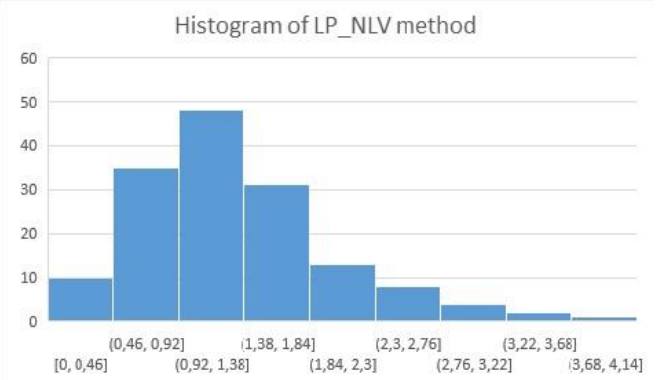

Figure 9. Histogram of LP_NLV method

To compare analytically the proposed method to other methods, we used the Analysis of variance (ANOVA) with dependent samples (dependence by image). The software $\mathrm{R}$ gives the following Anova table: 
Table 4. Anova table with one factor: method

\begin{tabular}{lclll}
\multicolumn{2}{c}{ Df } & Sum Sq & Mean Sq & F value $\operatorname{Pr}(>\mathrm{F})$ \\
Method & 9 & 25467 & 2829.6 & $742<2 \mathrm{e}-16 * * *$ \\
Residuals & 1341 & 5114 & 3.8 &
\end{tabular}

As $\operatorname{Pr}(>\mathrm{F})$ is smaller than $1 \%$, in table 4 ., the methods are significantly different. We used thenthe Newman Keuls test to compare the methods two-by-two and made groups having significantly the same mean. The software R shows the results of the test as follows:

Table 5. Test of Newman Keuls

\begin{tabular}{|c|c|c|}
\hline \multicolumn{3}{|c|}{ \$groups } \\
\hline & RMSE & groups \\
\hline SD & 12.6072900 & $\mathrm{a}$ \\
\hline BG & 11.0447767 & $\mathrm{~b}$ \\
\hline PCA & 8.7139600 & $\mathrm{c}$ \\
\hline DWT & 4.1941660 & d \\
\hline DCT_var & 2.8395233 & e \\
\hline GIF & 2.5146353 & e \\
\hline LP_DWT & 2.0496413 & f \\
\hline LP_PCA & 1.9954953 & $\mathrm{f}$ \\
\hline LP_NLV & 1.3446913 & $\mathrm{~g}$ \\
\hline NLV & 0.59215 & \\
\hline
\end{tabular}

From table 5., we have the means of RMSE of methods which are significantly different except the methods DCT_var and GIF form the group "e" and the methods LP_DWT and LP_PCA form the group "f".

The proposed method NLV has a smaller mean and significantly different of the all methods. We conclude that the proposed method is better than other methods.

\section{Conclusion}

This paper presents the image fusion method based on neighbour local variability (NLV). The principal method of fusion is described in details. The result of the experiment shows that the NLV method gives a significant improvement result in both visual and quantitative image fusion comparing to other fusion methods which are respectively DWT and LP-DWT. Laplacian pyramid with NLV as a selection rule was also applied, LP-NLV. Based on the experiment result, LP-NLV is better than LP-DWT and DWT.

The advantage of the proposed method lies in the fact that it takes into account the variability between each pixel and its neighbours. This gives a power to the coefficient of the pixel located in the focus part.This method can be extended to multimodal images used in particular in medicine (scanner, echography, X-ray, etc.) to give the presence of certain cancer cells seen in one image and not visible in another image. 
The method proposed can be used inmany applications such as:

1) Drone: it is a new technology in digital imaging, it hasopened up unlimited possibilities for enhancingphotography. Drone can capture images on the samescene that zooms in on different objects, and at variousaltitudes. It produces several images on the samescene but with different objects in-focus.

2) For quality control of food industry: cameras areused to take pictures. Each camera targets one ofseveral objects to detect an anomaly. The objects are on atreadmill. To have a photo containing all the objectsclearly, we can use the proposed method of fusion whichgives more details.

The perspectives of this work:

- As many work on image fusion, implementing grayscale images, all proposedmethods in this paper are performed on the grayscale image.However, these proposed methods can be extendedto color images as color conveys significantinformation.

- We are also encouraged to fuse more than twoimages by taking into account the local variabilityin each image (intra-variability) and variabilitybetween image (inter-variability). Inter-variability can detect the 'abnormal pixels' amongthe images.

\section{REFERENCES}

[1] Naidu, V.P.S. and. Raol, J.R. (2008) "Pixel-level Image Fusion using Wavelets and Principal Component Analysis", Defence Science Journal, Vol. 58, No. 3, pp. 338-352.

[2] Tian, J., Chen, L., Ma, L., Yu, W., (2011) "Multi-focus image fusion using a bilateral gradient-based sharpness criterion", Optic Communications, 284, pp 80-87.

[3] Zhan, K., Teng, J., Li, Q., Shi, J. (2015) "A novel explicit multi-focus image fusion method", Journal of Information Hiding and Multimedia Signal Processing, vol. 6, no. 3, pp.600-612.

[4] Mallat, S.G. (1989) "A Theory for multiresolution signal decomposition: The wavelet representation", IEEE Trans. Pattern Anal. Mach. Intel., 11(7), 674-93.

[5] Pajares, G., Cruz, J.M. (2004) "A Wavelet-Based Image Fusion Tutorial”, Pattern Recognition 37. Science Direct.

[6] Guihong, Q., Dali, Z., Pingfan, Y. (2001) "Medical image fusion by wavelet transform modulus maxima". Opt. Express 9, pp. 184-190.

[7] Indhumadhi, N., Padmavathi, G., (2011) "Enhanced Image Fusion Algorithm Using Laplacian Pyramid and Spatial Frequency Based Wavelet Algorithm", International Journal of Soft Computing and Engineering (IJSCE). ISSN: 2231-2307, Vol. 1, Issue 5.

[8] Sabre, R. Wahyuni, I.S, (2020) "Wavelet Decomposition and Alpha Stable", Signal and Image Processing (SIPIJ), Vol. 11, No. 1. pp. 11-24.

[9] Jinjiang Li , Genji Yuan and Hui Fan (2019) "Multifocus Image Fusion Using Wavelet-DomainBased Deep CNN", Computational Intelligence and Neuroscience, Vol. 2019 Article ID 4179397 | https://doi.org/10.1155/2019/4179397

[10] Burt, P.J., Adelson, E.H. (1983) "The Laplacian Pyramid as a Compact Image Code", IEEE Transactions on communication, Vol.Com-31, No 40.

[11] Burt, P.J. (1984) "The Pyramid as a Structure for Efficient Computation. Multiresolution Image Processing and Analysis", A. Rosenfeld, Ed., Springer-Verlag. New York.

[12] Burt, P.J., Kolezynski, R.J. (1993) "Enhanced Image Capture Through Fusion", in: International Conference on Computer Vision, pp. 173-182.

[13] Wang, W., Chang, F. (2011) "A Multi-focus Image Fusion Method Based on Laplacian Pyramid", Journal of Computers, Vol.6, No 12. 
[14] Zhao, P., Liu, G., Hu, C., Hu, and Huang, H. (2013) "Medical image fusion algorithm on the LaplacePCA”. Proc. 2013 Chinese Intelligent Automation Conference, pp. 787-794.

[15] Verma, S. Kaur K.,, Kumar M., R..(2016) "Hybrid image fusion algorithm using Laplacian Pyramid and PCA method", proceeding of the Second International Conference on Information and Communication Technology for Competitive Strategies.

[16] Wahyuni, I.S, Sabre, R. (2019) "Multifocus Image Fusion Using Laplacian Pyramid Technique Based on Alpha Stable Filter", CRASE Vol. 5, No. 2. pp. 58-62.

[17] Wahyuni, I.S, Sabre, R. (2016) "Wavelet Decomposition in Laplacian Pyramid for Image Fusion", International Journal of Signal Processing Systems Vol. 4, No. 1. pp. 37-44.

[18] Haghighat, M.B.A, Aghagolzadeh, A., Seyedarabi, H. (2010)“Real-time fusion of multifocus images for visual sensor networks". Machine vision and image processing (MVIP), 2010 6th Iranian. 2010.

[19] Bavirisetti, D.P., and Dhuli, R.(2016) "Multi-focus image fusion using multi-scale image decomposition and saliency detection", Ain Shams Eng. J., to be published. [Online]. Available: http://dx.doi.org/ 10.1016/j.asej.2016.06.011.

[20] Petland, A.(1984) "A new sense for depth of field", IEEE Transactions on Pattern Analysis and Machine Intelligent, Vol. 9, No. 4, pp. 523-531.

[21] Nayar, S.K.(1992) "Shape from Focus System", Proc. of IEEE Conf. Computer Vision and Pattern Recognition, pp. 302-308.

[22] Gonzales, R.C., Woods, R.E. (2002) "Ditigal Image Processing” 2nd edition. Prentice Hall.

[23] Liu,R., Li, Z., Jia, J.(2008) "Image Partial Blur Detection and Classification”, Computer Vision and Pattern Recognition, CVPR 2008. IEEE Conference DOI: 10.1109/CVPR.2008.4587465

[24] Aslantas, V. (2007) "A depth estimation algorithm with a single image." Optic Express, Vol. 15, Issue 8. OSA Publishing.

[25] Elder, J.H., Zucker, S.W.(1998) “ Local Scale Control for Edge Detection and BlurEstimation.” IEEE Transactions on Pattern Analysis and Machine Intelligence, Vol.20, No.7.

[26] www.rawsamples.ch. Accessed: 15 November 2017.

[27] Kumar, A., Paramesran, R., Lim, C. L., and Dass, S.C. (2016) "Tchebichef moment based restoration of Gaussian blurred images”. Applied Optics, Vol. 55, Issue 32, pp. 9006-9016.

\section{ANNEX 1}

Consider, without loss the generality that we have a focus pixel (x, y) in image I1 and blurred in image $\mathrm{I} 2$ as in Fig. 1.
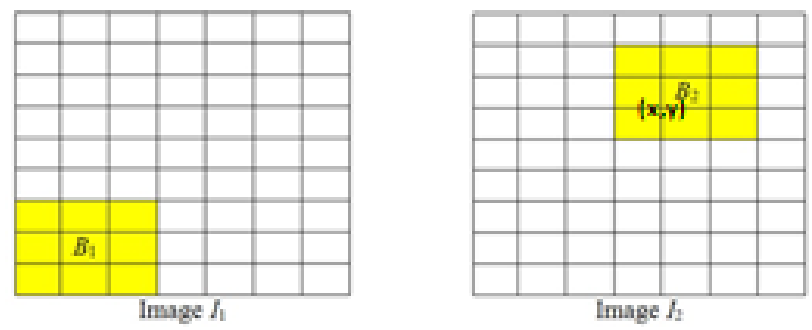

Fig. 1. Two multi-focus images, the yellow part is blurred area and the white part is clear (focus) area.

The neighbor local variability of images I1 and I2, respectively is defined in (1) by:

$v_{a, 1}(x, y)=\exp \left(\sqrt{\frac{1}{R} r_{1}(x, y)}\right)$ and $v_{a, 2}(x, y)=\exp \left(\sqrt{\frac{1}{R} r_{2}(x, y)}\right)$ where $r_{1}(x, y)$ and $r_{2}(x, y)$

can be written as follows: 


$$
\begin{aligned}
& r_{1}(x, y)=\sum_{m=0}^{2 a} \sum_{n=0}^{2 a}\left|I_{1}(x, y)-I_{1}(x+(m-a), y+(n-a))\right|^{2} \\
& r_{2}(x, y)=\sum_{m=0}^{2 a} \sum_{n=0}^{2 a}\left|I_{2}(x, y)-I_{2}(x+(m-a), y+(n-a))\right|^{2}
\end{aligned}
$$

Let $I_{R}$ is the reference image of multi-focus images $I_{1}$ and $I_{2}$. Moreover, it is shown in [20] and [21] that the blurred image can be seen as the product convolution between the reference image and Gaussian filter:

$$
I_{1}(x, y)=\left\{\begin{array}{ll}
w_{1}^{*} I_{R}(x, y), & (x, y) \in B_{1} \\
I_{R}(x, y), & (x, y) \notin B_{1}
\end{array} I_{2}(x, y)= \begin{cases}w_{2} * I_{R}(x, y), & (x, y) \in B_{2} \\
I_{R}(x, y), & (x, y) \notin B_{2}\end{cases}\right.
$$

where $w_{1}$ and $w_{2}$ are Gaussian filter defined by:

$$
\begin{gathered}
w_{1}(k, l)=w_{1}(k, l)=\frac{\exp \left(-\frac{k^{2}+l^{2}}{2 \sigma_{1}{ }^{2}}\right)}{\sum_{k=-s_{1}}^{s_{1}} \sum_{l=-s_{1}}^{s_{1}} \exp \left(-\frac{k^{2}+l^{2}}{2 \sigma_{1}}\right)},(\mathrm{k}, 1) \in\left[-s_{1}, s_{1}\right]^{2}, \\
w_{2}(k, l)=\frac{\exp \left(-\frac{k^{2}+l^{2}}{2 \sigma_{2} 2}\right)}{\sum_{k=-s_{2}}^{s_{2}} \sum_{l=-S_{2}}^{s_{2}} \exp \left(-\frac{k^{2}+l^{2}}{2 \sigma_{2}{ }^{2}}\right)},(k, l) \in\left[-s_{2}, s_{2}\right]^{2}
\end{gathered}
$$

The product convolution is defined as follows:

$$
\begin{aligned}
& w_{1} * I_{R}(x, y)=\sum_{k=-s_{1}}^{s_{1}} \sum_{l=-s_{1}}^{s_{1}} w_{1}(k, l) I_{R}(x-k, y-l), w_{2} * I_{R}(x, y)=\sum_{k=-s_{2}}^{s_{2}} \sum_{l=-s_{2}}^{s_{2}} w_{2}(k, l) I_{R}(x-k, y-l), \\
& \text { Put } r_{1}(x, y)=\sum_{m=0}^{2 a} \sum_{n=0}^{2 a}\left|D_{(m, n)}^{1}(x, y)\right|^{2} \quad r_{2}(x, y)=\sum_{m=0}^{2 a} \sum_{n=0}^{2 a}\left|D_{(m, n)}^{2}(x, y)\right|^{2} \\
& \text { where } D_{(m, n)}^{1}(x, y)=I_{1}(x, y)-I_{1}(x+(m-a), y+(n-a)) \\
& D_{(m, n)}^{2}(x, y)=I_{2}(x, y)-I_{2}(x+(m-a), y+(n-a))
\end{aligned}
$$

\section{Proposition:}

The local variability on blurred part is smaller than the local variability on focused part. Let $(x, y) \in B_{2}$ (the blurred part of $\mathrm{I}_{2}$ ) and $(x, y) \notin B_{1}$ (focus par of $\mathrm{I}_{1}$ ), then $\left(r_{2}(x, y) \leq r_{1}(x, y)\right)$.

\section{Proof:}

For that, we use Plancherel theorem: 


$$
\sum_{m=0}^{2 a} \sum_{n=0}^{2 a}\left|D_{(m, n)}^{1}(x, y)\right|^{2}=\frac{1}{(2 a+1)^{2}} \sum_{\omega_{m=0}}^{2 a} \sum_{n=0}^{2 a}\left|\widehat{D}_{(n, m)}^{1}(x, y)\right|^{2}(8)
$$

where $\widehat{D}_{(n, m)}^{1}(x, y)$ is Fourier transform of $D_{(m, n)}^{1}(x, y)$.

$$
\widehat{D}_{(n, m)}^{1}(x, y)=F T\left[D_{(m, n)}^{1}(x, y)\right]=F T\left[I_{1}(x, y)-I_{1}(x+(m-a), y+(n-a))\right]
$$

As $(x, y) \in B_{2}$ therefore $(x, y) \notin B_{1}$, from (4), equation (9) can be written as follows:

$\widehat{D}_{(n, m)}^{1}(x, y)=F T\left[I_{R}(x, y)-I_{R}(x+(m-a), y+(n-a))\right]$

and

$$
I_{2}(x, y)=\sum_{k=-s_{2}}^{s_{2}} \sum_{l=-s_{2}}^{s_{2}} w_{2}(k, l) * I_{R}(x-k, y-l)
$$

By using the definition of convolution, equation (11) can be written as:

$$
I_{2}(x, y)=\sum_{k=-\infty}^{\infty} \sum_{l=-\infty}^{\infty} w_{2}(k, l) 1_{\left[-s_{2}, s_{2}\right]^{2}} I_{R}(x-k, y-l)(12)
$$

and

$$
I_{2}(x, y)=\left(w_{2} 1_{\left[-s_{2}, s_{2}\right]^{2}}\right) * I_{R}(x, y)(13)
$$

Where

$$
1_{\left[-s_{2}, s_{2}\right]^{2}}(k, l)=\left\{\begin{array}{rr}
1, & \text { if }(\mathrm{k}, \mathrm{l}) \in\left[-s_{2}, s_{2}\right]^{2} \\
0, & \text { otherwise }
\end{array}\right.
$$

The Fourier transform of $D_{(m, n)}^{2}(x, y)$ is

$$
\begin{gathered}
\widehat{D}_{(n, m)}^{2}(x, y)=F T\left[w_{2} 1_{\left[s_{2}, s_{2}\right]^{2}} * I_{R}(x, y)-w_{2} 1_{\left[s_{2}, s_{2}\right]^{2}} * I_{R}(x+(m-a), y+(n-a))\right] \\
=F T\left[w_{2} 1_{\left[s_{2}, s_{2}\right]^{2}} *\left(I_{R}(x, y)-I_{R}(x+(m-a), y+(n-a))\right)\right] \\
=F T\left[w_{2} 1_{\left[s_{2}, s_{2}\right]^{2}}\right] F T\left[I_{R}(x, y)-I_{R}(x+(m-a), y+(n-a))\right]
\end{gathered}
$$

Substitute (10) into (14), we get 


$$
\begin{gathered}
\widehat{D}_{(n, m)}^{2}(x, y)=F T\left[w_{2} 1_{\left.\left[s_{2}, s_{2}\right]^{2}\right]} \widehat{D}_{(p, q)}^{1}(x, y)\right. \\
=\left(\sum_{k=-\infty}^{\infty} \sum_{l=-\infty}^{\infty} w_{2}(k, l) 1_{\left[s_{2}, s_{2}\right]^{2}}(k, l) e^{-i 2(k p+l q)}\right) \widehat{D}_{(n, m)}^{1}(x, y)
\end{gathered}
$$

Hence, from equation (15), we can obtain

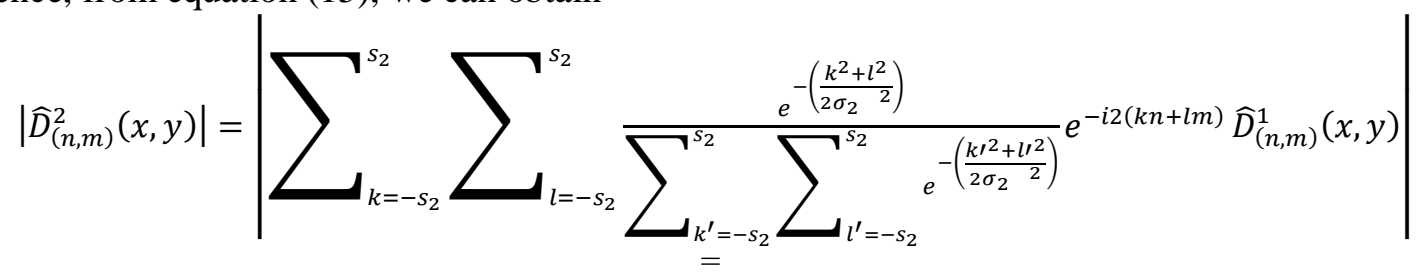

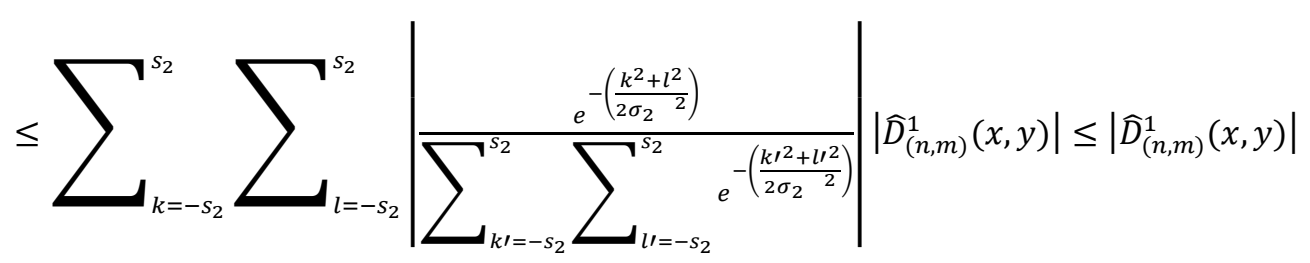

(16)

On the other hand, from equation (5) and Plancherel-Parseval's theorem, we have

$$
r_{2}(x, y)=\sum_{m=0}^{2 a} \sum_{n=0}^{2 a}\left|D_{(m, n)}^{2}(x, y)\right|^{2}=\frac{1}{(2 a+1)^{2}} \sum_{m=0}^{2 a} \sum_{n=0}^{2 a}\left|\widehat{D}_{(n, m)}^{2}(x, y)\right|^{2}
$$

From (16), we get

$$
r_{2}(x, y) \leq \frac{1}{(2 a+1)^{2}} \sum_{m=0}^{2 a} \sum_{n=0}^{2 a}\left|\widehat{D}_{(p, q)}^{1}(x, y)\right|^{2} \leq \sum_{r_{2}(x, y) \leq r_{1}(x, y)}^{2 a} \sum_{n=0}^{2 a}\left|\widehat{D}_{(m, n)}^{1}(x, y)\right|^{2}
$$

This proves that the local variability in blurred part is smaller than local variability in focused part.

\section{AuThORS}

Rachid Sabre received the PhD degree in statistics from the University of Rouen, Rouen, France, in 1993 and Habilitation (HdR) from the University of Burgundy, Dijon, France, in 2003.He joined Agrosup Dijon, Dijon, France, in 1995, where he is an Associate Professor. From 1998 through 2010, he served as a member of "Institut de Mathématiques de Bourgogne", France. He was a member of the Scientific Council AgroSup Dijon from 2009 to 2013. In 2012, he has been a member of " Laboratoire Electronique, Informatique, et Image” (Le2i), France. Since 2019 has been a member of Laboratory Biogeosciences UMR CNRS, University of Burgundy. He is author/co-author of numerous papers in scientific and 
technical journals and conference proceedings. His research interests lie in areas of statistical process and spectral analysis for signal and image processing.

Ias Sri Wahyuni was born in Jakarta, Indonesia, in 1986. She earned the B.Sc. and M.Sc. degrees in mathematics from the University of Indonesia, Depok, Indonesia, in 2008 and 2011, respectively.In 2009, she joined the Department of Informatics(COMPUTING) System, Gunadarma University, Depok, Indonesia, as a Lecturer. She is currently a $\mathrm{PhD}$ student at University of Burgundy, Dijon, France. Her current research interests include statistics and image processing.

(C) 2020 By AIRCC Publishing Corporation. This article is published under the Creative Commons Attribution (CC BY) license. 